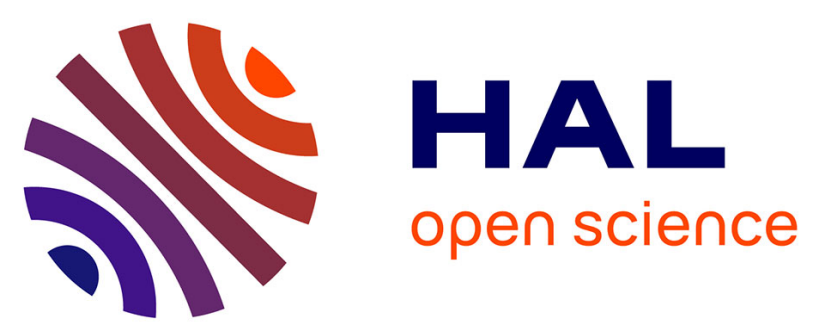

\title{
ÉTUDE DE BIFURCATIONS DE GUIDES D'ONDES PAR MÉTHODE MODALE, APPLICATION AUX SILENCIEUX RÉACTIFS ET AUX INSTRUMENTS DE MUSIQUE À VENT
}

J. Kergomard, A. Khettabi

\section{To cite this version:}

J. Kergomard, A. Khettabi. ÉTUDE DE BIFURCATIONS DE GUIDES D'ONDES PAR MÉTHODE MODALE, APPLICATION AUX SILENCIEUX RÉACTIFS ET AUX INSTRUMENTS DE MUSIQUE À VENT. Journal de Physique IV Proceedings, 1992, 02 (C1), pp.C1-85-C1-88. 10.1051/jp4:1992114 . jpa-00251097

\author{
HAL Id: jpa-00251097 \\ https://hal.science/jpa-00251097
}

Submitted on 1 Jan 1992

HAL is a multi-disciplinary open access archive for the deposit and dissemination of scientific research documents, whether they are published or not. The documents may come from teaching and research institutions in France or abroad, or from public or private research centers.
L'archive ouverte pluridisciplinaire HAL, est destinée au dépôt et à la diffusion de documents scientifiques de niveau recherche, publiés ou non, émanant des établissements d'enseignement et de recherche français ou étrangers, des laboratoires publics ou privés. 


\title{
ÉTUDE DE BIFURCATIONS DE GUIDES D'ONDES PAR MÉTHODE MODALE, APPLICATION AUX SLENCIEUX REACTIFS ET AUX INSTRUMENTS DE MUSIQUE À VENT
}

\author{
J. KERGOMARD et A. KHETTABI
}

Laboratoire d'Acoustique, URA 1101 CNRS, Université du Maine, Route de Laval, BP. 535, F-72017 Le Mans cedex, France

\begin{abstract}
The shape of an equivalent electrical circuit for a guide with side branch is obtained using modal theory in matricial formalism, in a general system of coordinates.
\end{abstract}

\section{Position du problème}

Le problème de la bifurcation de guides d'ondes acoustiques a déjà été étudié par divers auteurs, utilisant différentes méthodes (MILES [1], LAPIN [2], COTTE [3], KEEFE [4]). Nous proposons une méthode très simple et générale en étendant la solution au problème de deux jonctions. Nous utilisons la méthode matricielle décrite en [5], et cherchons les matrices reliant (à basse fréquence) les modes plans entre eux.

Considérons le problème d'un guide (principal) sur lequel se greffe un autre guide, $A$, à angle droit (fig.1). Nous considérons l'équation intégrale de Helmholtz-Huygens dans la cavité hachurée où il est supposé ne pas exister de sources:

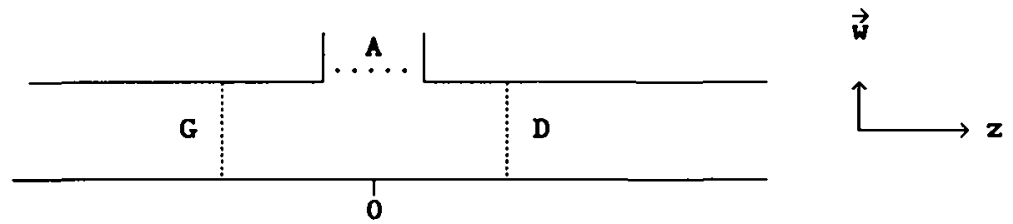

Fig. 1 : géomètrie de la bifurcation

$$
p(\vec{r})=\int_{S_{A}+S_{G}+S_{D}}\left[G\left(\vec{r}^{,} \vec{r}^{\prime}\right) \partial_{n}, p\left(\vec{r}^{\prime}\right)-\partial_{n^{\prime}}, G\left(\vec{r}, \vec{r}^{\prime}\right) p\left(\vec{r}^{\prime}\right)\right] d S^{\prime}
$$

où $p$ est la pression acoustique, $G$ la fonction de Green dans la cavité considérée. Le principe du calcul est de projeter l'éq.1 sur les modes propres des deux guides sur $S_{{ }^{\prime}}, S_{G}, S_{D^{\prime}}$ en décomposant pression et vitesse sur ces surfaces de la façon suivante :

$$
p(\vec{r})={ }^{t} \psi(w) P(z) \quad \text { et } \quad v(\vec{r})=\frac{1}{S}^{t} \psi(w) U(z)
$$


$\vec{w}$ est le vecteur (bldimensionnel) caractérisant un point courant d'une tranche d'abscisse $z$. $\psi$ est le vecteur mode propre du guide, S la section. Si on choisit la fonction de Green correspondant à la cavité fermée, seul le premier terme de l'intégrale subsiste, et on obtlent une relation du type impédance entre un vecteur pression et un vecteur vitesse (triplement infinis). Cette solution, efficace dans le cas d'une jonction quelconque entre trols guides, est moins bonne dans le cas présent : nous préférons choisir la fonction de Green du tube principal infini, ce qui évite une sommation. Alors le second terme de l'intégrale ne s'annule que sur $S_{A}$. En notation matricielle, la fonction de Green s'écrit :

$$
G\left(r, r^{\prime}\right)=\frac{1}{2 j \omega \rho} \quad t \psi(w) z_{c} E^{-}(z) E^{+}\left(z^{\prime}\right) \psi\left(w^{\prime}\right) \quad \text { si } z^{\prime}>z
$$

où $Z_{c}, E^{-}$et $E^{+}$sont des matrices diagonales: $Z_{c i j}=\frac{\rho c}{S} \frac{k}{k_{1}} \delta_{i j}$ et

$$
E_{1 j}^{ \pm}(z)=e^{\mp j k_{1} z} \delta_{1}, k_{1} \text { est le nombre d'onde du mode } 1 \text { dans la direction } z
$$

Un point essentiel est que dans l'équation (1) les intégrales correspondant a $S_{G}$ et $S_{D} S^{\prime}$ annulent $S^{\prime} i l n^{\prime} y$ a pas de sources à gauche et à droite des surfaces $S_{G}$ et $S_{D}$, respectivement (condition de Sommerfeld). S'il y a des sources, seul le champ venant de gauche, ou de droite respectivement, subsiste. Ainsi, si on écrit le champ de pression dans le guide à gauche de la bifurcation sous la forme suivante:

$$
p(\vec{r}))^{t} \psi(w)\left[E^{+}(z) P_{G}^{+}+E^{-}(z) P_{G}^{-}\right],
$$

l'intégrale correspondant à $S_{G}$ sera simplement ${ }^{t} \psi(w) E^{+}(z) P_{G}^{+}$, d'où:

$$
p(\vec{r})=\int_{S_{A}} G\left(\vec{r}, \vec{r}^{\prime}\right) \partial_{n}, p\left(r^{\prime}\right) d S^{\prime}+t_{\psi(w) E^{+}(z) P_{C}^{+}+t_{\psi(w) E^{-}}(z) P_{D}^{-}} .
$$

\section{Projection sur les modes}

La projection sur les modes sur $S_{A}, S_{C}, S_{D}$ donne alors :

$$
\left\{\begin{array}{l}
P_{G}^{-}=-\frac{1}{2} z_{C}{ }^{t} Q^{+} U_{A}+P_{D}^{-} ; P_{D}^{+}=-\frac{1}{2} z_{C}{ }^{t} Q^{-} U_{A}+P_{G}^{+} \\
P_{A}=-Z_{A A} U_{A}+Q^{+} P_{G}^{+}+Q^{-} P_{D}^{-}
\end{array}\right.
$$

où $Q^{ \pm}=\frac{1}{S_{A}} \int_{S_{A}} \psi_{A}{ }^{t} \psi E^{ \pm}(z) d S$ et $z_{A A}=\frac{j \omega P}{S_{A}^{2}} \int_{S_{A}} \int_{S_{A}} \psi_{A}(r) G^{t} \psi_{A}\left(r^{\prime}\right) d S d S^{\prime}$. 
$\mathrm{P}_{\mathrm{G}}^{+}$et $\mathrm{P}_{\mathrm{D}}^{-}$sont les sources du problème. Si la discontinuité $\mathrm{n}^{\prime}$ est pas découplée à d'autres discontinuítés, c'est-à-díre si la distance à d'autres discontinuités (ou sources) est assez grande, seuls les modes propagatifs subsistent (mode plan à basse fréquence), et le système (5) se simplifie. On décompose d'abord dans le guide principal entre mode plan et modes supérleurs et on réarrange les trois équations, on obtlent alors:

$$
\left\{\begin{array}{l}
p_{G}-p_{D}=j z_{C} t_{\beta} U_{A} ; u_{G}-u_{D}=t_{\alpha} U_{A} \\
p_{A}=-H U_{A}+\alpha\left(p_{G}+p_{D}\right) / 2-j z_{C} \beta\left(u_{G}+u_{D}\right) / 2
\end{array}\right.
$$

où $\quad \alpha=\frac{1}{S_{A}} \int_{S_{A}} \cos k z \psi_{A} d S, \quad \beta=\frac{1}{S_{A}} \int_{S_{A}} \sin k z \psi_{A} d S, \quad z_{c}=z_{c 00}$

La matrice $H$ est obtenue a partir de $Z_{A \Lambda}$ en remplaçant $G$ par sa partie réelle. Les quantités minuscules correspondent au mode plan pour la section (fictive) $z=0$. Si le guide A est passif, sa matrice impédance d'entrée est connue: l'impédance des modes évanescents est l'impédance caractéristique, celle du mode plan, $z_{0}$, dépendant de l'impédance terminale:

$$
z_{A}=\left(\frac{z_{0}}{z_{C A}^{\prime}}\right) \text {. D'où } u_{A}=\left(Z_{A}+H\right)^{-1}\left[\alpha\left(p_{C}+p_{D}\right) / 2-j z_{c} \beta\left(u_{C}+u_{D}\right) / 2\right] \text {. }
$$

La solution du système 6 peut être trouvée soit par méthode variationnelle (résultat approché cf [4]), solt par inversion de matrice, soit par itération (cf [5]).

\section{Circuit électrique équivalent}

II reste à décomposer $P_{A}$ et $U_{A}$ sur les modes plan et supérieurs du guide A pour trouver la relation entre modes plans. On obtient ainsi le résultat cherché. Dans le cas (assez général) où le guide $A$ est symétrique par rapport au plan $z=0$, les modes sont soit symétriques(s), solt antisymétriques $(a)$, et sont découplés dans les matrices $Z_{A}, H$, $\alpha$ et $\beta$ :

$$
\alpha=\left(\frac{\alpha_{s}}{0}\right) ; \beta=\left(\frac{0}{\beta}\right) ; \quad z_{A}+H=\left(\frac{Z_{A s}+H_{s}}{O} \mid \frac{0}{Z_{A a}+H_{a}}\right)
$$

De plus, on peut séparer le mode plan (0) des autres modes symétriques

$$
\alpha_{s}=\left(\begin{array}{c}
\alpha_{0} \\
\alpha_{s}^{+}
\end{array}\right) ; \quad Z_{A s}+H_{s}=\left(\frac{z_{0}+h_{0}}{h_{s}} \mid \frac{t_{h}}{Z_{A s}^{\prime}+H_{s}^{\prime}}\right)
$$


On obtient alors le schéma équivalent de la figure 2 :

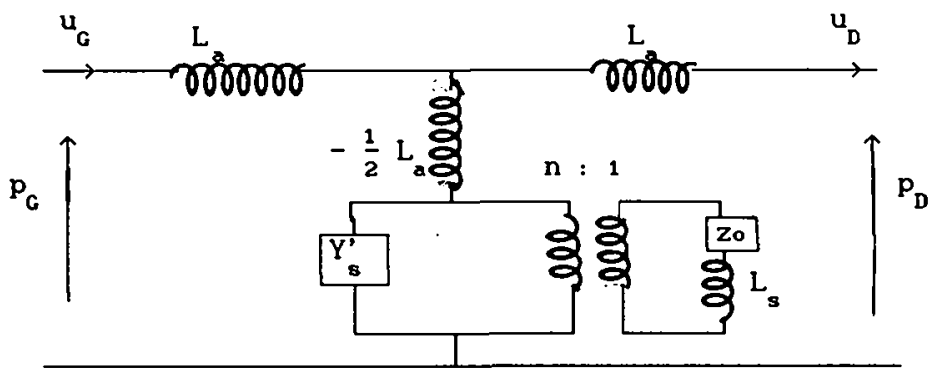

Fig.2: Schéma équivalent à une bifurcation

où

$$
\begin{aligned}
& j \omega L_{a}=z_{c}^{2} t_{\beta_{a}}\left(Z_{A a}+H_{a}\right)^{-1} \beta_{a} ; j \omega L_{s}=h_{0}-t_{h_{s}}\left(Z_{A s}^{\prime}+H_{s}^{\prime}\right)^{-1} h_{s} ; \\
& n=\alpha_{0}-t \alpha_{s}^{\prime}\left(Z_{A s}^{\prime}+H_{s}^{\prime}\right)^{-1} h_{s} \text {, et tend vers } 1 \text { quand } \omega \longrightarrow 0 .
\end{aligned}
$$

Enfín, $Y_{S}^{\prime}={ }^{t} \alpha_{s}^{\prime}\left(Z_{A s}^{\prime}+H_{s}^{\prime}\right)^{-1} \alpha_{s}^{\prime}$, proportionnel à $\omega^{3}$, ne peut pas être négligé quand la branche parallèle résonne. Ce circuit falt apparaitre des termes quí jusque là n'avaient pas été mis en évidence: ainsi, le terme ' $Y^{\prime}$ ', qui peut avoir une importance pour les maximums de transmission d'un résonateur branché. A noter que le circuit est valable pour des trous latéraux d'instrument à vent, qu'ils solent ouverts ou fermés.

\section{Conclusion}

Même si on ne calcule pas les termes ainsi obtenus (peu de géométries le permettent simplement), la forme du circuit est intéressante. Nous avons généralisé cette technique à des guides avec deux bifurcations, ou à deux guides reliés par un guide commun, dont un cas particulier est un silencieux à tubes perforés. Si deux guides sont reliés par la surface $S_{A}$, on écrit 2 systèmes (5) pour chacun des guides, on élimine $P_{A}$ et on obtient la matrice $(4 \times 4)$ reliant les modes plans. On peut ainsi montrer que la théorie classique (SULLIVAN [6]) des silencieux à tubes perforés oublie l'effet des modes antisymétriques.

Nous remercions Douglas KEEFE pour de fructueuses discussions.

\section{Références}

[1] MILES, J.W., J. Acoust. Soc. Am. 19 (1947) 572-579.

[2] LAPIN, A.D., Soviet Phys. Acoust. 7 (1961) 77-79.

[3] COTTE-DUNAJEWSKI, T., Contribution à l'étude des résonateurs, Thèse de spéclallté en acoustique, Aix-Marseille I, (1977).

[4] KEEFE, D. H. , J. Acoust. Soc. Am. 72 (1982) 676-687).

[5] KERGOMARD, J., J. Acoust Ique 4 ((1991), 111-138.

[6] SUllivaN, J.W., J. Acoust. Soc. Am. 66 (1979) 772. 